\title{
A possible Universal Origin of Hadronic Cosmic Rays from Ultrarelativistic Ejecta of Bipolar Supernovae
}

\author{
R.Plaga* \\ Max-Planck-Institut für Physik (Werner-Heisenberg-Institut) \\ Föhringer Ring 6 \\ D-80805 München, Germany \\ Received 27 September 2001; received in revised form 3 April 2002; accepted 3 \\ April 2002 \\ Communicated by R.P.Kirshner
}

\begin{abstract}
Based on the "cannonball model" for gamma-ray bursts of Dar and De Rújula it is proposed that masses of baryonic plasma ("cannonballs"), ejected in bipolar supernova explosions in our Galaxy are the sources of hadronic Galactic cosmic rays (CRs) at all energies. The propagation of the cannonballs in the Galactic disk and halo is studied. Two mechanisms for the acceleration of the observed CRs are proposed. The first is based on ultrarelativistic shocks in the interstellar medium and could accelerate the bulk of CRs up to the "knee" energy of $4 \times 10^{15} \mathrm{eV}$. The second operates with second-order Fermi acceleration within the cannonball. If the total initial energy of the ejected plasmoids in a SN explosion is $10^{53}$ ergs or higher this second mechanism may explain the CR spectrum above the knee up to the highest observed energies. It is shown that together with plausible assumptions about CR propagation in the Galactic confinement volume the observed spectral indices of the CR spectrum can be theoretically understood to first order. The model allows a natural understanding of various basic CR observations like the absence of the Greisen-Zatsepin cutoff, the anisotropy of arrival directions as function of energy and the small Galactocentric gradient of the CR density.
\end{abstract}

\section{Introduction}

\subsection{Motivation for a "unified" (single-source class) explanation of the spectrum of Galactic cosmic rays}

The flux of the dominating hadronic component of the local non-solar cosmic rays (CR), has been measured between about $0.1 \mathrm{GeV}$ and $3 \cdot 10^{20} \mathrm{eV}$. Neglecting solar modulation

*Telephone 49-89-32354-224; FAX: 49-89-3226704; email:plaga@mppmu.mpg.de 
effects, its energy spectrum can be well described by a single power-law that changes its slope slightly at only two energies - at $\approx 4 \cdot 10^{15} \mathrm{eV}$ (the "knee") and $\approx 3 \cdot 10^{18} \mathrm{eV}$ (the "ankle"). This striking simplicity and unity of the data over more than 10 decades in energy originally led most authors to ascribe the origin of hadronic cosmic rays to a single "universal" source class.

In an influential review, Ginzburg[31] suggested that the remnants of supernova explosions in our Galaxy (SNRs) are the dominant source of CRs at all energies. However, SNRs cannot explain the origin of CRs with energies above above about $10^{18} \mathrm{eV}$ because at these energies charged particles are certainly no longer confinable in the relatively weak magnetic fields of SNRs. In another classical paper Burbidge 10] proposed the radical alternative of an extragalactical origin of CRs at all energies. In this scenario it proved difficult to understand the origin of CRs with low energies: no extragalactic source class seems able to fill up the universe with the rather high total energy-density of the locally observed CRs $\left(\approx 0.5 \mathrm{eV} / \mathrm{cm}^{3}\right)$ within a Hubble time.

As a compromise, an "eclectic scenario" for CR origin was proposed by Morrison[49]. This idea became widely accepted in the 1970s in the following form: SNRs accelerate the CRs below the knee and extragalactic sources are the source of CRs above the ankle. Between the knee and ankle another class of objects - of an as yet unclear nature - is supposed to accelerate the CRs?. However, the ankle lies just at the energy where CRs become unconfined from the Galaxy. This fact can only be causally understood if the same universal source class supplies the cosmic rays below and above the ankle [66, 53]. The eclectic scenario was born of distress, namely of the difficulty that no "universal" source class was known that can accelerate particles to the highest observed energies and at the same time supply the rather high local energy density of CRs. The aim of this paper is to revisit the idea of a universal origin of Galactic CRs and to propose a source class capable of accelerating nuclei to the observed energy spectrum.

\subsection{Clues to the nature of the "single source" class from experimental evidence on cosmic rays}

In all probability the universal source class resides within or near to our Galaxy. It is all but impossible to supply the high ambient Galactic cosmic-ray energy density with any extragalactic source class. The absence of the Greisen cutoff in the experimentally determined cosmic-ray spectrum is another strong argument in favour of a Galactic source class. The observed lack of an overall anisotropy of arrival directions in UHE cosmic rays, the very small anisotropy at all smaller energies, and the - at best - very weak concentration of the cosmic-ray density towards the Galactic centre forces us to conclude that the "universal source class" (if it exists) has a very different spatial distribution than SNRs which

\footnotetext{
${ }^{1}$ Possible further small deviations are discussed in section 2.2 .1

${ }^{2}$ Biermann's 河 theory of CR origin does not make this assumption and is further discussed in section 1.4
} 


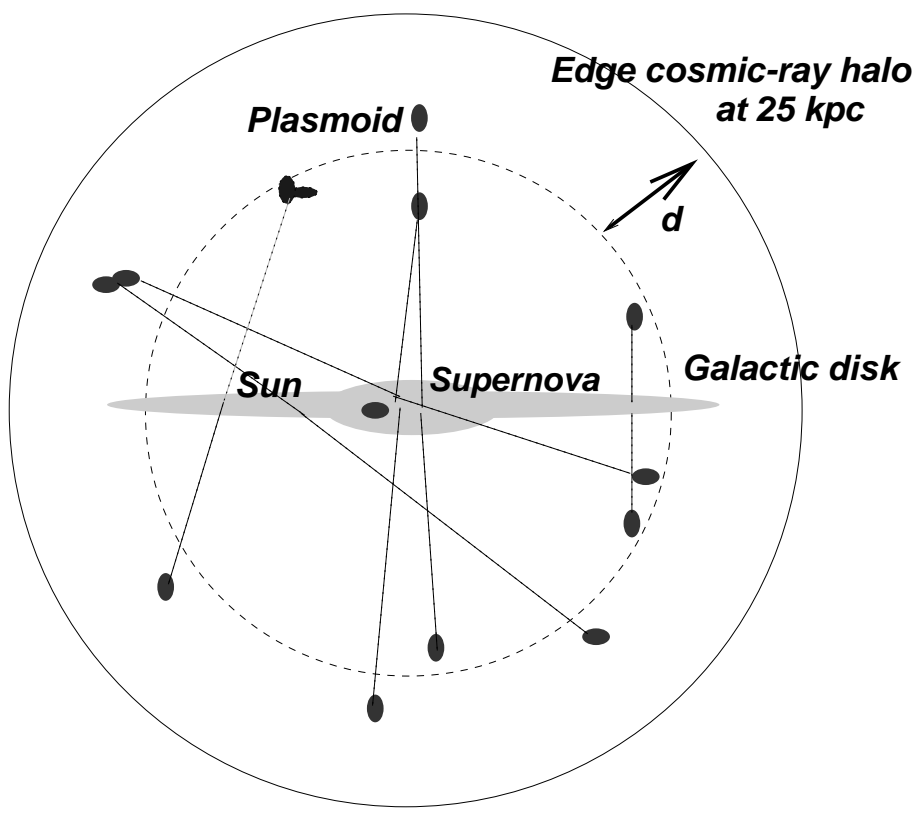

Figure 1: A scheme of the scenario proposed here. Energetic core-collapse supernovae in the Galactic disk and centre eject plasmoids ("cannonballs") that are slowed down to nonrelativistic speeds in the Galactic halo. These cannonballs are proposed to accelerate the major part of hadronic CRs at all energies.

populate the disk and central region of the Galaxy. It seems to reside in the Galactic halo. A possible origin of CRs at all energies in the Galactic halo, due to Fermi acceleration in hydromagnetic turbulence, has been discussed by Burbidge [9]. Biermann \& Davis [6] and Plaga 53] discuss mechanisms by which a large Galactic halo may be filled homogeneously with CRs.

\subsection{Ejecta from bipolar supernovae as sources of Galactic cosmic rays}

There has been recently strong observational evidence that core-collapse supernovae (SNe) can produce "long" $\gamma$-ray bursts (GRBs) [40]. A related recent revolution in the understanding of SNe is observational evidence that apparently all core-collapse SNe are asymmetric "bipolar" explosions 69. Observations cannot yet directly decide yet if:

a. only very few "exceptional" SNe emit GRBs[51] or

b. a sizeable fraction of or even all core-collapse SNe emit GRBs jet like into a narrow cone along a symmetry axis 11.

There is direct observational evidence that GRBs are emitted by matter at ultrarelativistic speeds. Circumstantial evidence suggests that GRBs involve rather strong magnetic fields. This suggests that they are excellent candidates for the acceleration of CRs at all energies. Especially if option b. above should be correct, the following question will be decisive for CR physics: 


\section{Do the well known non-relativistic isotropic ejecta (SNRs) or the only recently discovered relativistic jet-like ejecta dominate the CR production of all super- novae?}

It has been suggested that the very high ejection speeds in conjunction with strong magnetic fields allow acceleration to much higher energies than SNRs 68, 70. This makes jet-like SN ejecta serious candidates for the long sought universal source class of hadronic CRs at all energies [17, 19].

The potential importance of Gamma-Ray bursters for the origin of Galactic CRs at energies above the knee had been first studied by Milgrom and Usov 48 and Dar 17]. Recently Pugliese et al. 56] concluded that GRBs are unimportant as sources of CRs at all energies whereas Dermer 22] argues that objects related to GRBs might supply most of the observed low-energy hadronic CRs. The reason for this disagreement is the use of very different GRB models.

\subsection{Aims and plan of this paper}

The basic idea of the present paper - that ultrarelativistic ejecta from stellar-collapse events accelerate the dominant part of hadronic CRs at all energies and release it in the Galactic halo (fig. 1) - was first proposed by Dar \& Plaga 19]. At that time the nature of the GRB emitting collapse-events was unclear (supernovae were only mentioned as one possibility) and they were called "Galactic Gamma-Ray Bursters (GGRBs)". In the present paper the nature of these collapse events, the propagation of their ejecta and the CR-acceleration mechanisms operating near them are discussed more quantitatively.

Section 2 discusses what one can conclude about the properties of a hypothetical "singlesource class" purely from the phenomenology of CRs. I conclude that universal sources must be mainly located outside the solar circle, in the Galactic halo.

Further observational evidence in favour of such a location of the CR sources is discussed in section 3. The Galacto-centric distribution of $\gamma$-rays and anisotropy of charged CRs support the idea that CRs below the ankle have their origin mainly (but not exclusively) at Galacto-centric distances exceeding the solar one.

A candidate for the "single-source class" is proposed in section ㄴ:t ultrarelativistic plasmoids ejected in bipolar supernova explosions ("cannonballs" 20]). The motion of cannonballs in the Galactic disk and halo is discussed. Section 5 studies mechanisms with which these ejecta produce the hadronic CR spectrum at all energies (2).

This scenario is related to ideas of Bierman[]] who proposes an origin of Galactic CRs up to the ankle in SNRs and an origin of the higher energy CRs in extragalactic jets. My work can be seen as a proposal to "replace" the non-relativistic SN remnant with ultrarelativistic supernova ejecta. These ejecta ("cannonballs") "assume" the role of an extragalactic jet in a later stage of their evolution.

The ideas of sections 2 and 4 are logically independent. It is conceivable that there is 
a single-source class, but I err in its identification, or that cannonballs contribute to the CR spectrum only in certain limited energy ranges. Section 6 confronts expectations in a scenario where cannonballs dominate the CR production at all energies with observations. Section 1 summarises the main assumptions, achievements and predictions of this paper. This paper only treats hadronic CRs, leaving aside the origin of electrons in the Galactic CRs as a completely separate issue.

\section{Properties of a hypothetical single-source class of cosmic rays derived from observations}

In this section I try to derive the likely properties of a hypothetical "universal" CR-source class from observed CR properties without making assumptions about the physical nature of the sources. In this I optimistically take it for granted that a "simple" understanding of the CR spectrum - e.g. without accidental cancellations of additional effects - is possible.

\subsection{Energy dependence of cosmic-ray diffusion coefficient assumed in this work}

In this work I will assume that the cosmic-ray propagation is purely diffusive. The dependence of the diffusion coefficient $\mathrm{D}$ on the total energy per nucleus $\mathrm{E}$ is usually parametrised as:

(1) $\quad D \sim E^{\alpha}$

There are two theoretically motivated values for $\alpha$. A plasma with turbulent inhomogeneities following a "Kolmogoroff spectrum" would lead to a diffusive CR motion with $\alpha=0.33$, alternatively, with a "Kraichnan spectrum" $\alpha=0.5$ would apply 12. Direct determinations of the electron-density fluctuations in the interstellar medium [1] indicate a turbulence spectrum that leads to a power-law dependence of D according to eq.(1) up to very high energies 5 . However, such measurements are not precise enough to determine $\alpha$. $\mathrm{CR}$ data obtained at low energies indicate $\alpha \approx 0.6-0.7[5]$. This value can be made compatible with both theoretical values by postulating varying degrees of "re-acceleration" during the propagation of cosmic rays. There is recent experimental evidence for some re-acceleration [16] but it is not yet clear which value for $\alpha$ is preferred by these data.

It is generally accepted and plausible that CRs are magnetically confined to the Galaxy up to energies of about $\mathrm{E}_{\text {ankle }} \approx 3 \times 10^{18} \mathrm{eV}[5]$. At lower energies the power-law index of the CR spectrum changes only once by 0.3 at the knee. In principle the "knee" could signal a change from $\alpha=0.33$ to $\alpha=0.5$. However, this is unlikely because the observed change in the power-law index of $\approx 0.3$ is significantly larger than the difference between the theoretically preferred indices of $(0.5-0.33)$. I conclude that in any simple scenario - where $\alpha$ has one

\footnotetext{
${ }^{3}$ up to scales corresponding to the Larmor radius of $\approx 10^{17} \mathrm{eV}$ particles in the Galactic magnetic field.
} 
of the theoretically preferred values at all energies - $\alpha$ is constant at either 0.33 or 0.5 at all energies up to the ankle. In the next section I will argue that - at least in a scenario with "universal sources" - $\alpha=0.5$ is the preferred value.

\subsubsection{An argument in favour of $\alpha=0.5$ at all energies valid under the assump- tion that universal sources of CRs exist (can be omitted on first reading)}

Let $d$ be the typical distance of the universal sources from the edge of the Galactic confinement volume (see fig.(1)). The CR flux at an energy of

$\mathrm{E}_{1}=40 \mathrm{GeV}$ is a factor $\mathrm{K}=\left(\mathrm{E}_{1} / \mathrm{E}_{\text {ankle }}\right)^{-\alpha}$ higher than at energy $\mathrm{E}_{\text {ankle }}$ due to a $\mathrm{K}$ times longer confinement time $\tau$. The confinement time at energy $E_{1}$ is given as $\tau_{1} \approx \mathrm{d}^{2} / \mathrm{D}_{1}$, where $\mathrm{D}_{1}$ is the diffusion coefficient at energy $\mathrm{E}_{1}$. For a halo size $\mathrm{h} \approx 20 \mathrm{kpc}$ (a large halo size is preferred for for the universal-source scenario, see section 2.3.2) D was determined from measured isotope-ratios as approximately $9 \times 10^{28} \mathrm{~cm}^{2} / \mathrm{sec} 63$. The confinement time at energy $\mathrm{E}_{\text {ankle }}$ is approximately $\tau_{a} \approx \mathrm{d} / \mathrm{c}$, the value for unconfined motion. Setting $\mathrm{K}=\tau_{1} / \tau_{a}$ I obtain:

$$
\begin{array}{r}
d \approx\left(E_{1} / E_{\text {ankle }}\right)^{-\alpha} \times D_{1} / c \\
\approx 8 \mathrm{kpc} \text { for } \alpha=0.5 \\
\approx 0.3 \mathrm{kpc} \text { for } \alpha=0.33
\end{array}
$$

The confinement volume is certainly extended by more than $10 \mathrm{kpc}$ in our scenario (see section 2.3.2) and therefore the case $\alpha=0.33$ would lead to a "crowding" of the universal sources in the outer $<3 \%$ of the confinement volume. This seems rather unnatural and we therefore assume in the following that $\alpha=0.5$ at all energies $<E_{\mathrm{a}}$.

To summarise, the assumption of a $\mathrm{E}^{0.5}$ dependence of $\mathrm{CR}$ diffusion at all energies where Galactic confinement occurs is theoretically motivated, in agreement with all data and allows a natural spatial distribution of universal sources.

\subsection{The value of the spectral indices of the source spectrum, before Galactic modulation( $[19])$}

The assumption of $\alpha=0.5$ (eq.11) fixes the spectrum of the "universal CR sources". Let the source spectrum be given as:

(3) $\quad F_{\text {source }} \sim E^{\delta}$

If this source emits into a volume that diffusively confines CRs the observed spectrum within this volume is given as:

(4) $\quad F_{\text {observed }}\left(E<E_{\text {ankle }}\right) \sim E^{(\delta+\alpha)} \sim E^{\gamma_{2}}$

\footnotetext{
4 The diffusion coefficients are specified by Strong \& Moskalenko (1998) at a rigidity of 5 GV. For intermediate masses $(A=16)$ this corresponds to an energy per nucleus $\mathrm{E}_{1} \approx 40 \mathrm{GeV}$.
} 
This spectrum holds at all energies where cosmic rays are magnetically confined to the Galaxy (i.e. up to the ankle). At higher energies the pure source spectrum is expected again:

(5) $\quad F_{\text {observed }}\left(E>E_{\text {ankle }}\right) \sim E^{\delta} \sim E^{\gamma_{3}}$

at higher energies. The observed index $\gamma_{2}$ between knee and ankle is $\left(\delta_{2}+\alpha\right)=-3$. I infer for the source index $\delta_{2}=-2.5$, a value that is compatible within $1.3 \sigma$ with world-mean of experimental determinations of the index above the ankle of $\gamma_{3}=-2.75 \pm 0.2[50$ ] as it must be within my model. According to section 2.1 I assume no breaks in the energy dependence of the diffusion coefficient which governs CR propagation, so the "knee" in the CR spectrum must be a source feature. The spectral index of the source must then decrease by 0.3 at the knee, so the $\delta_{1}=-2.2$ at energies below the knee. This general scenario for the origin of the total CR spectrum is illustrated in fig.(3) (taken from Ref.[19]).
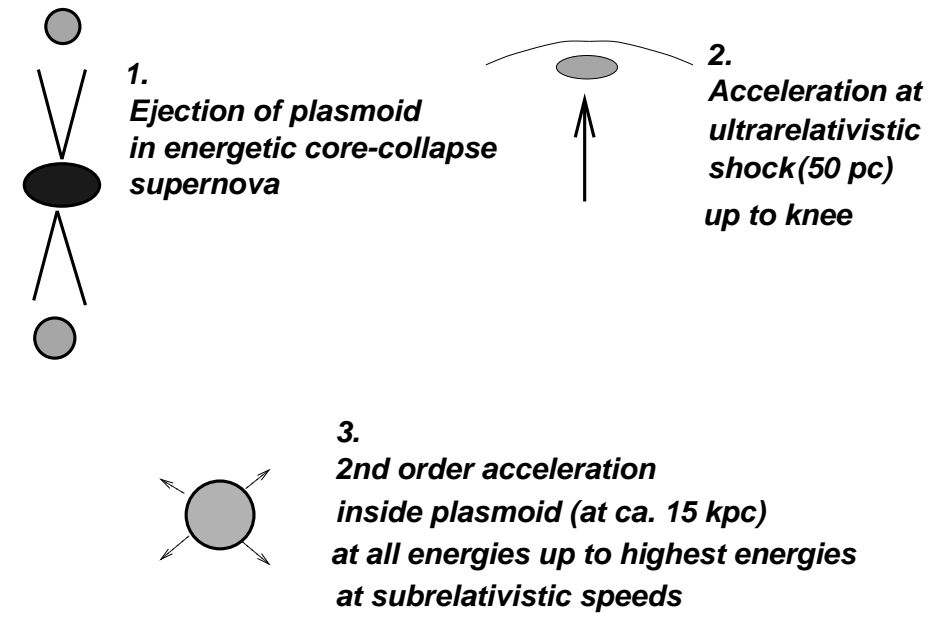

Figure 2: A schematic sketch of the main steps in the scenario discussed here. The shaded balls symbolise the cannonballs. The thin un-arrowed line in the second image stands for a shock wave driven by the cannonball into the interstellar medium. Step 1 is further explained in section 4.1, step 2 in section 5.1 and step 3 in section 5.2 .

\subsubsection{Unsettled issues: structure at the "knee" and the "second knee"}

The discussion of the spectrum in the previous section is oversimplified. Erlykin and Wolfendale [26] have argued that an additional component that raises the flux by about a factor 2 in a narrow energy interval near the knee at $4 \times 10^{15} \mathrm{eV}$ is indicated by the data. Detailed models for the "universal sources" have to deal with this feature.

At energies around $4 \times 10^{17} \mathrm{eV}$ the spectrum steepens by about 0.2 in the power law index, thus forming a "second knee" [50]. In our scenario it seems natural that the transition between diffusion in a turbulent plasma and approximate straight-line motion is gradual 
and therefore a steepening occurs before the ankle.

I defer more detailed speculations about the nature of these finer details of the spectrum to a later time.

\subsection{Spatial location of the universal CR sources}

\subsubsection{Upper limit on distance of universal sources from Galactic centre: iso- topic data of low-energy CRs}

The data on isotopic composition of CRs yield an upper limit on the size of the Galactic halo in which magnetic confinement takes place. Because the CR spectrum can only arise in the way outlined in section 2.2 if the particles are initially magnetically confined, the maximal size of the halo is also an upper limit on the distance of the universal CR sources from the Galactic centre.

The size h of a cosmic-ray halo around the Galaxy is only weakly constrained by results on the abundance of unstable isotopes in low-energy cosmic rays 63. A halo with $\mathrm{h}>4 \mathrm{kpc}$ is required to exist, and $\mathrm{h} \gg 20 \mathrm{kpc}$ seems to be ruled out with all parameter choices, so we assume a maximal allowed size of $30 \mathrm{kp}$ 䑤.

\subsubsection{Lower limit on distance of universal sources from Galactic centre: isotropy of UHE CRs}

No significant anisotropies have been found in the sky distribution of CRs with energies above $4 \times 10^{19} \mathrm{eV}[50$, 73]. However, with a world statistics of 114 events, anisotropies on the order of up to $30 \%$ amplitude cannot be ruled out. At these energies - far above the "ankle" - light nuclei are expected to move in straight lines to good approximation in our scenario. Also light nuclei comprise a major fraction of all CRs in "universal scenario". (fig. 8). We conclude that a major part $(>50 \%)$ of all universal sources must lie at distances beyond the solar circle (i.e. Galacto-centric distances $>10 \mathrm{kpc}$ ).

\subsubsection{The likely location of "universal" CR sources}

The above consideration indicate a typical location of the "universal sources" at about 10 - $30 \mathrm{kpc}$ from the Galactic centre. For the reasons mentioned above these limits (especially the upper one) are quite uncertain. A detailed simulation using the code of Strong \&

\footnotetext{
5 This upper limit is quite uncertain mainly for four reasons. 1. Strong and Moskalenko[63] do not take into account uncertainties of the input parameters, which are considerable. 2. The spatial distribution of universal CR sources is expected to be different from the one assumed by Strong and Moskalenko. 3. CR-electron sources might have a very different spatial distribution (following the SNR distribution) from the one of CR-hadron sources (following the cannonball distribution in our scenario). 4. The plausible possibility of a diffusion coefficient that is smaller in the Galactic disk than the halo 32 is not considered. This possibility gains in potential importance if sources are not located in the Galactic disk.
} 
Moskalenko 63 and taking into account the special features of the universal source class is urgently required.

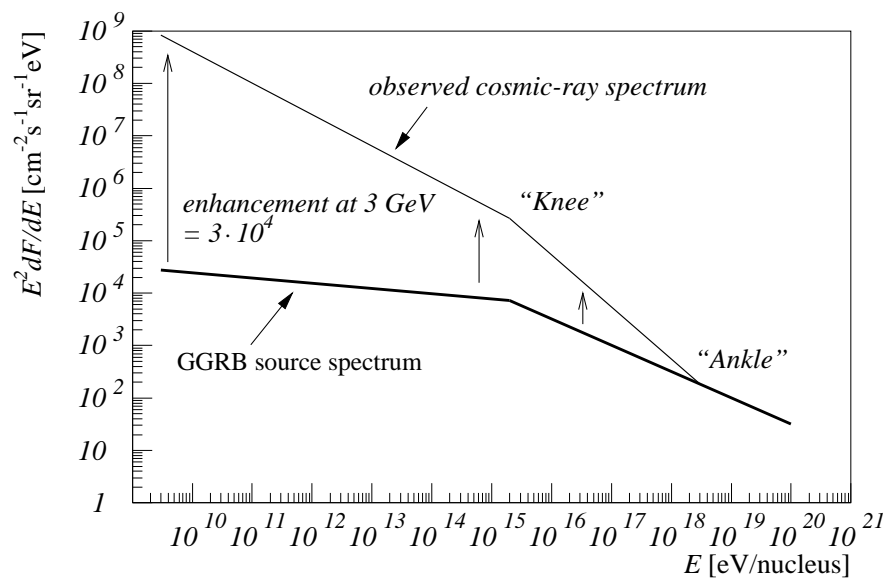

Figure 3: The observed flux of cosmic rays (thin line) as a function of primary energy $\mathrm{E}$ is well described by a power law that changes its slope sharply at only two energies, the "knee" and the "ankle". At energies below the ankle it is enhanced (by a factor $\left(\mathrm{E} / \mathrm{E}_{\text {ankle }}\right)^{-0.5}$ ) over the Galactic Gamma-Ray Burster (GGRB) source spectrum (thick line, a power law with differential power law index of -2.2 below the knee and $\simeq-2.5$ above it) by way of trapping in the Galactic halo magnetic fields. The "GGRBs" are identified with bipolar SNe in the present paper.

\section{Observational evidence in favour of CR sources located mainly (but nor completely) outside the solar circle}

\subsection{Galacto-centric gradient of cosmic rays from $\gamma$-ray data}

The analysis of EGRET data from Mattox and Strong 62] and Erlykin et al. [25] find that the cosmic-ray density is constant within about $25 \%$ between 5 and $15 \mathrm{kpc}(5-18 \mathrm{kpc}$ in Erlykin et al. 25]) distance from the Galactic centre. In this range the SNR- and pulsardensities density decrease by about a factor 5 to 10 . Were these object classes the main sources of CRs - and taking into account CR diffusion in a large halo - a much larger decrease of CR density by a factor $\geq 3$ over this range of Galacto-centric distances would be expected [8]. The last bin (15 to $30 \mathrm{kpc}$ in Mattox and Strong[62]) shows a density about $30 \%$ lower than the previous ones. The analysis of the EGRET team[37] shows a drop in the density by about $30 \%$ in the range $5-10 \mathrm{kpc}$ than a rise in density up to $15 \mathrm{kpc}$ in 3 quadrants. At larger Galacto-centric distances a precipitous drop in density was inferred. 
These results are not in complete agreement with each other but they do suggest that the universal sources of Galactic CRs are mainly located in shell at about $20 \mathrm{kpc}$ distance from the Galactic centre. In this approximation one expects a constant density at smaller distances and a rapid drop at larger distances; this is in agreement with the data.

\subsection{Anisotropy of arrival directions of Galactic cosmic rays}

If all universal CR sources were located outside the solar circle one would expect no anisotropy of Galactic cosmic rays at any energy: there is none inside a shell isotropically emitting cosmic rays. There is however experimental evidence for small anisotropies in Galactic cosmic rays at all energies 44]. The observationally well established energyindependent anisotropy with an amplitude of $\approx 8 \times 10^{-4}$ at energies between about 1 and $100 \mathrm{TeV}$ is probably the result of a motion of the local rest frame of cosmic rays relative to the solar system with a speed of about $80 \mathrm{~km} / \mathrm{sec}$ via the Compton-Getting effect 15]. The nature of this motion is unclear. Schmele 60 recently proposed an anomalous velocity component of the solar-system surroundings connected with a motion of the "local fluff" out of the centre of the local super-bubble in which the CRs are at rest. If this interesting suggestion is correct, the anisotropy below $10^{14} \mathrm{eV}$ contains information about the plasma that confines CRs but holds no direct clues about the origin of cosmic rays.

At higher energies there is a large world-data set which indicates an anisotropy that increases with energy $\mathrm{E}$ as $\mathrm{E}^{0.5}$ up to the highest energies (fig. 1) 44. In spite of a very large systematic scatter, the measurements of the direction of first-harmonic maximum intensity in the total world-data set seem to cluster around the Galactic-centre direction (see fig.5). Because individual anisotropy measurements usually have marginal statistical significances and the derived locations of maximum intensity do not agree well, doubts have been expressed if these results are more than upper limits on a possible anisotropy. However, a recent measurements and reanalysis indicate significances for anisotropies above $5 \sigma$.

Clay et al.[14] reanalysed the world data set in the southern hemisphere in the energy range $10^{15}-3 \cdot 10^{15} \mathrm{eV}$ and found an anisotropy amplitude of

(6) $\delta_{a}=0.33 \pm 0.06 \%$

which corresponds to a $5.5 \sigma$ effect. The direction of maximum intensity has a right ascension of $19.7 \pm 0.7 \mathrm{~h}$, not far from the one of the Galactic centre (at $17.8 \mathrm{~h}$ ). Hayashida et al. [33] report an anisotropy at energies above $10^{18} \mathrm{eV}$ of $3.9 \pm 1 \%$ with a statistical significance of $5.4 \sigma$. Again an excess mainly from the Galactic-centre direction is indicated. These two high-significance results are in reasonable agreement with each other if the $\mathrm{E}^{0.5}$ energy dependence of the anisotropy amplitude of the world data set is assumed. A more detailed future analysis needs to take into account the different geographical locations of the various experiments which necessitate small corrections of the measured anisotropy amplitudes and directions. 


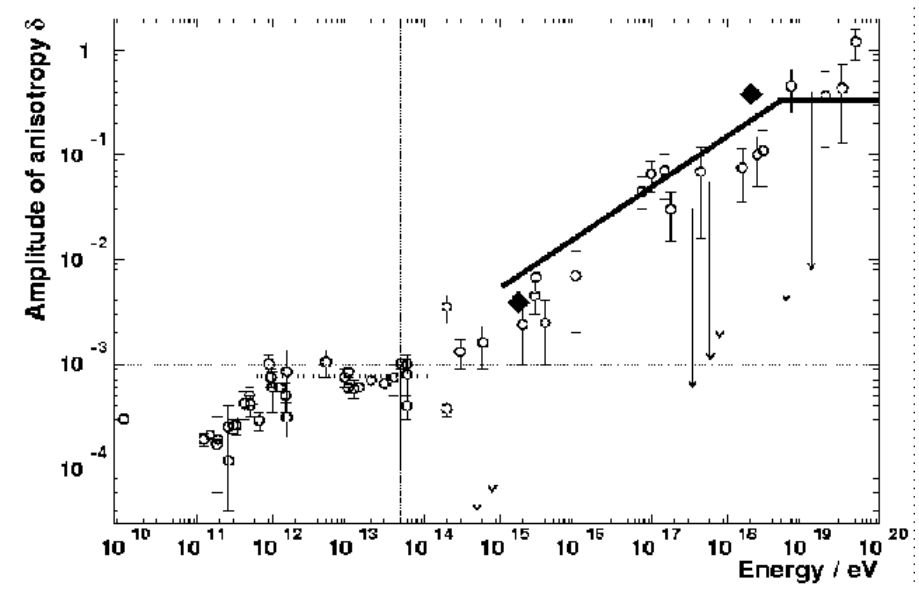

Figure 4: World-data set as of 1997 of measurements the anisotropy amplitude of the first harmonic as a function of energy. From the thesis of Schmele 60 where all references are given. The thin dotted lines indicate the data point obtained in this thesis from data obtained by the HEGRA air-shower array. The diamonds indicate the recent results by Clay et al.[14] (near $1 \mathrm{PeV}$ ) and Hayashida et al. [33] (near $1 \mathrm{EeV}$ ), discussed in the text. The quoted errors of these two determinations are smaller than the symbol sizes. The thick, full line indicate the anisotropy theoretically predicted (eq.(7) with $f=0.15$ (section 6.2.1)). The thick dotted line indicates the constant anisotropy at energies between about 1-100 $\mathrm{TeV}$ probably due to an anomalous motion of the solar system relative to the rest frame of CRs (see text).

Summarising, two new high significance measurements confirm the long known indication of an anisotropy amplitude rising with square root of energy and prefer an origin of this anisotropy of arrival directions from the direction of the Galactic centre.

A natural origin for the observed anisotropy is a net outward-streaming motion of cosmic rays produced in the Galactic centre region. If such a streaming motion exists, the Compton-Getting effect 15 leads to a large scale anisotropy with a maximal intensity near the Galactic centre. Let us estimate the expected magnitude an anisotropy $\delta_{a}$ produced in this way. The preferred values of the energy dependence of the diffusion constant D, halos size $\mathrm{h}$ and spectral index $\gamma$ as discussed in sections 2.1.1 and 2.3 were chosen. Here " $f$ " is the fraction of $\mathrm{CR}$ sources that are located within the solar circle.

$$
\begin{gathered}
\delta_{a}=0.5 \%\left(D / 9 \cdot 10^{28} \mathrm{~cm}^{2} / \mathrm{sec}\right) /(h / 20 k p c) / c \times \\
((\gamma / 2.7)+1)(f / 0.1) \cdot\left(\left(E / 10^{15} \mathrm{eV}\right) / 40 G e V\right)^{0.5}
\end{gathered}
$$

This expression is valid from about $10^{15} \mathrm{eV}$ (at lower energies another source of anisotropy dominates) to about $3 \times 10^{18} \mathrm{eV}$ (where CRs become unconfined to the Galaxy). Here " $f$ " is the fraction of CR sources that are located within the solar circle. Eq. (7) is plotted in fig. A and is seen to agree with the data within the rather large errors. 


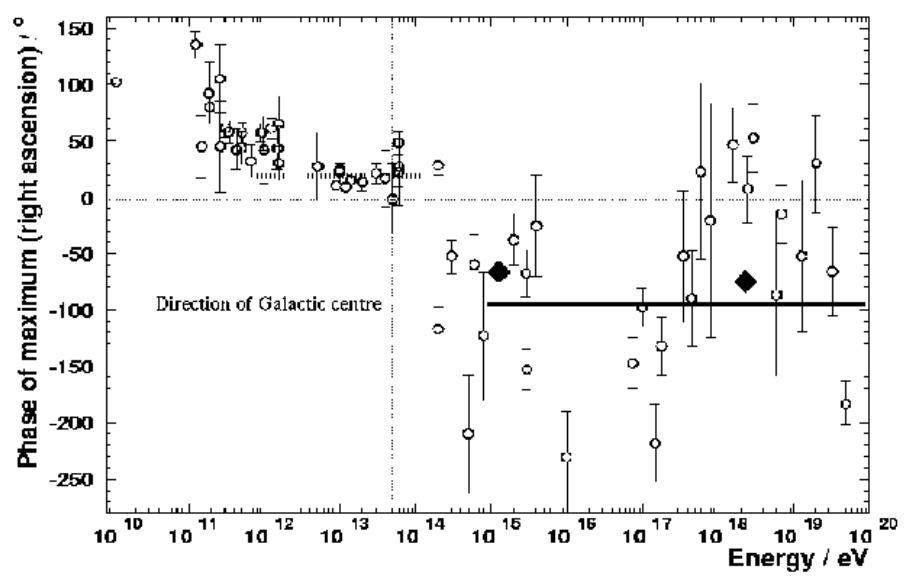

Figure 5: World-data set as of 1997 of measurements the direction of the maximum of the CR intensity in right ascension as a function of energy (first harmonic). From the thesis of Schmele[60] where all references are given. The thin dotted lines indicate the data point obtained in this thesis from data obtained from the HEGRA air-shower array. The thick, full line indicates the direction towards the Galactic centre. The diamonds indicate the recent results by Clay et al. 14] (near $1 \mathrm{PeV}$ ) and Hayashida et al. 33] (near $1 \mathrm{EeV}$ ) discussed in the text. The error bars of the result of Clay et al. (1998) are comparable to the symbol size, no error was given in Hayashida et al.[33] for the determination of the direction. The thick dotted line indicates the range where an energy-independent anisotropy was found (see text).

A consistent explanation of the $C R$ anisotropies at all energies is thus possible if $f \approx 10 \%$ of the universal sources are located in the Galactic centre and $90 \%$ outside the solar circle. This is consistent with the observational constraints of section 2.3 and the prediction within the cannonball model (section 6.2.1).

\section{A candidate for the "universal" CR sources: ultrarelativis- tic ejecta from bipolar supernovae (aka "cannonballs")}

\subsection{Ultrarelativistic ejecta ("cannonballs") from supernovae}

\subsubsection{The cannonball model of Gamma-Ray Bursts}

Recently Dar and De Rújula 20, 21] developed the "cannonball model" for Gamma-Ray Bursts (GRBs). Similar to Woosley's 75] "failed supernova" concept it proposes that the mechanism for core-collapse supernovae can involve a phase where matter falls onto a central compact object from large distances. Episodic accretion onto this central black hole or neutron star leads to the subsequent repeated emission of distinct masses of plasma,the "cannonballs" (fig.2), initially moving with a Lorentz $\Gamma \approx 1000$. Radiation emitted by the 
cannonballs is quantitatively shown to explain the properties of GRBs. This radiation is emitted isotropically in the rest frame of the cannonball, but in the observer frame the Lorentz boost strongly collimates the radiation into a cone with opening angle $\theta=1 / \Gamma$ along the direction of motion ("relativistic beaming"). Therefore only a very small fraction $\approx \theta^{2} / 4 \approx 2 \times 10^{-7}$ of all cannonballs are visible.

Recently I found [54 that it is possible to quantitatively understand a "cepheid-like" relationship between variability and total luminosity of GRBs 27] and between the width and height of spikes within a given GRB [58] within the cannonball model. This makes it plausible that the broad features of the cannonball model are correct. I will assume that this is the case in the rest of this paper.

The initial energy of these cannonballs is not well known. If the core of a pre-collapse star is rapidly rotating, $\approx 3 \cdot 10^{53}$ erg may be stored as rotational energy of the collapsed object, and the jet seems the most effective way to dispose off this energy [52]. I will assume below a total energy of $10^{53} \mathrm{erg}$ ejected in each of the symmetric cannonball jets in a SN (rather higher than what is normally assumed but in line with the expectations of Dar and De Rújula 21]). Smaller values lead to conditions in which CRs cannot be accelerated to the observed ultra-high energies (section 5.2).

\subsubsection{The frequency of supernovae that eject cannonballs, inferred from the observed GRB rate}

For some GRBs Fenimore and Ramirez-Ruiz 27] infer redshifts in excess of 12 from their variability - luminosity relation. Because it seems unplausible that much larger redshifts occur, within the cannonball model this indicates that cannonballs ejected exactly into the observer direction are visible from the entire universe. If that is true, the rate of cannonball-ejecting supernovae in our Galaxy is given as [18]:

$$
\begin{array}{r}
R_{\text {prog }}=\frac{1}{50 \text { years }} \frac{R_{\mathrm{GRB}}}{10^{3} / \text { year }} \times \\
\left(1 /\left(\frac{\left(\theta_{\text {mean }}^{2} / 5\right)}{4(\Gamma / 1000)^{2}}\right)\right) \times \\
\left(\left(L_{B}(\text { Galaxy }) / 2.3 \times 10^{10} L_{\odot}\right) /\right. \\
\left.\left(\rho_{B} / 1.8 h 10^{8} L_{\odot} \mathrm{Mpc}^{-3}\right)\right) / \\
\left(R_{\mathrm{SFR}(\mathrm{z}=0)} /\left(\int(1+z)^{-1} R_{\mathrm{SFR}(\mathrm{z})}\left(d V_{c} / d z\right) d z\right)\right) \times 15
\end{array}
$$

Here $R_{\mathrm{GRB}}$ is the observed total rate of GRBs 28], $\mathrm{L}_{B}$ is the B-luminosity of our Galaxy, $\rho_{B}$ is the B-luminosity density in the local universe [47, $\mathrm{R}_{\mathrm{SFR}}$ is the star formation rate at a respective redshift derived from optical observations 42. The preferred value was derived with a volume element $\mathrm{d} V_{c}$ for $\Omega=1$ and $\Lambda=0$. $\theta_{\text {mean }}^{2}$ is the mean squared angle between an observed GRB and the observer divided by $\Gamma^{2}$. This angle is expected to be somewhat larger than $1 / \Gamma^{2}$ because nearby GRBs are bright enough for detection also at 
viewing angles $>1 / \Gamma$. The mean $\left(\theta^{2}\right) \approx 5$ was inferred by calculating the $\theta^{2}$ for all bursts in Fenimore and Ramirez-Ruiz 27] using relations valid in the cannonball model [54]. An isotropic luminosity at $\theta=0$ of $2 \times 10^{55} \mathrm{erg} / \mathrm{sec}$ (the highest luminosity of any GRB in their sample) was assumed for this estimate. The preferred value of $R_{\text {prog }}=1 / 50$ years corresponds to the estimated total rate of all core-collapse supernova in our Galaxy [59], thus indicating that all core-collapse supernovae eject cannonballs.

The major uncertainty in the estimate of $R_{\text {prog }}$ is the dependence on the star-formation rate (SFR) on z. In particular Fenimore and Ramirez-Ruiz 27 derive a $R_{\text {SFR-GRB }}$ based on observations of GRB space densities alone. This SFR continues to rise at redshifts beyond 1 , in contrast to results based on optical observations. Using these results I obtain $R_{\mathrm{SFR}-\mathrm{GRB}(\mathrm{z}=0)} / \int(1+z)^{-1} R_{\mathrm{SFR}-\mathrm{GRB}(\mathrm{z})}\left(d V_{c} / d z\right) d z \approx 250$. This corresponds to $R_{\mathrm{prog}} \approx$ $1 / 800$ years. The possible choice of other world-models introduces another uncertainty of about a factor 2 in this estimate, so the plausible range of rates of cannonball-producing $\mathrm{SNe}$ is about $1 / 50-1 / 1600$ years. Preferred candidates for jet-forming core-collapse events have similar formation rates: $\mathrm{SN} I b / \mathrm{c}$ supernovae $(\mathrm{R} \approx 1 / 300$ years 59 ), "failed supernovae" ( $\mathrm{R}$ $\approx 1 / 1000$ years 75 ) and "collapsars" $(\mathrm{R} \approx 1 / 1500$ years 29 ).

Summarising, based on the observed GRB rate, the progenitor rate for cannonball ejecting supernova could lie between about 0.05 - 1 of total core-collapse SN rate.

\subsubsection{The total cannonball-energy converted into CRs}

The "CR luminosity" $\mathrm{L}_{\mathrm{cr}}$ - the energy per time in form of hadronic CRs injected into the Galactic confinement volume - is given as:

$$
\begin{array}{r}
L_{\text {cr }}=2 \times 10^{42} \mathrm{erg} / \sec \left(R_{\text {prog }} / 1000 \text { years }\right) \times \\
\left(E_{\text {tot }} /\left(2 \times 10^{53} \mathrm{erg}\right)\right)(\epsilon / 0.33)
\end{array}
$$

Here $\epsilon$ is the conversion efficiency of total energy to hadronic CRs within the collimated (section 4.2) cannonball, that is likely near the equipartition value of $1 / 3$ in our scenario (see section 5.2). $R_{\text {prog }}$ is the rate of cannonball-ejecting supernovae in our Galaxy. If cannonballs are the dominating source of hadronic CRs they must supply the observed "CR luminosity" $\mathrm{L}_{\mathrm{cr}}(\exp ) \approx 10^{41} \mathrm{erg} / \mathrm{sec}$ which can be inferred from experimental data[5]. Within the plausible range for $R_{\text {prog }}$ discussed in the previous section, less than $1 / 40$ of the total cannonball-energy has to be converted the energy of CR particles to explain the observed CR luminosity $\mathrm{L}_{\mathrm{cr}}$ (eq.(9)) .

\subsection{Collimation of the cannonball}

\subsubsection{Assumption of cannonball confinement}

In the model of Dar and De Rújula 20] the cannonball initially expands with the speed of sound in a relativistic gas $\mathrm{c} / \sqrt{3}$. It is a basic hypothesis of the present paper that some collimation mechnism prevents any further expansion of the cannonball once the cannonball has 
reached a limiting radius $R_{\text {limit }} \approx 0.3 p c$. If there is equipartition between magnetic-field energy density, turbulent plasma motion and cosmic-ray energy density, a total magnetic energy of $\mathrm{E}_{\text {tot }} / 3 \approx 3 \times 10^{52} \mathrm{erg}$ (see sect. 4.1.1) corresponds to a magnetic field inside the cannonball of $\mathrm{B}=\sqrt{E_{\text {tot }}} / \mathrm{R}_{\text {limit }}^{3} \approx 0.6 \mathrm{G}$.

Efficient collimation is commonly observed in extragalactic jets (for a review see[38]). There is no complete theoretical understanding of the mechanisms leading to collimation in general. Longair recently wrote 45]: "By which means relativistic jets are collimated, is one of the major unsolved problems of astrophsics. Almost certainly, the mechanism will require the presence of magnetic fields...". In principle a slight deviation from equipartition with strong magnetic fields might serve to contain turbulent plasma and CRs. However, the "plasma-virial theorem" forbids a purely magnetic stress balance without additional outer stresses [46]. The only viable candidate for the outer stress in the case of cannonballs seems to be ram pressure from the ambient interhalo medium. It has been shown long ago that ram pressure can confine a spherical mass of plasma 23], and Heinz recently argued that the same mechanism might lead to confinement of "bullets" ejected by GRBs 35. Equating the typical expected ram pressure onto a subrelativistic $\mathrm{CB}$ [ $\mathrm{P}_{\mathrm{ram}}=\mathrm{m}_{p}\left(\mathrm{n}_{\text {interhalo }} /\left(10^{-3} / \mathrm{cm}^{-3}\right)\right)$ $(\Gamma / 1.2) \mathrm{c}^{2}$ with its internal magnetic-field pressure $\mathrm{B}_{\text {int }}^{2} / 4 \pi$ shows that with the parameters indicated above ram pressure can only balance an internal field $B_{\text {int }}$ of about $10 \mathrm{mG}$, about a factor 50 smaller than required. Efficient collimation therefore requires a magnetic field that increases towards the interior of the CB (similar to what happens in a tokamak). What magnetic-field configuration of the cannonball would allow a stable confined configuration in conjunction with external ram pressure is an open question, closely related to Longair's "open problem" mentioned above. As a detailed simulation of the internal dynamics is beyond the scope of the manuscript, below I make the simplest possible assumption of free expansion until a limiting radius $\mathrm{R}_{\text {limit }}$ is reached. When the cannonball has slowed down to nonrelativistic speeds and the ram-pressure becomes very small, a final Sedov-Taylor expansion must take place 20].

\subsection{Motion of confined plasmoids in the Galaxy}

\subsubsection{The interstellar medium ambient to cannonballs}

Lingenfelter, Higdon and Ramaty [43] have pointed out that about $85 \%$ of all core-collapse supernovae are expected to occur in "super-bubbles". These are cavities in the interstellar medium (ISM) blown by repeated SN explosions and filled with hot $\left(10^{6} \mathrm{~K}\right)$ and tenuous (number density $10^{-3} / \mathrm{cm}^{3}$ ) gas. Their conclusion seems compelling to me. It is an undisputed observational fact that about $50 \%$ of the ISM volume of the Galactic disk is filled with super bubbles [61]. Therefore at least half of all supernovae are expected to occur in super bubbles. The actual fraction must be somewhat higher than this because

\footnotetext{
${ }^{6}$ see discussion in section 5.2 of the CB propagation
} 
core-collapse supernovae are known to be spatially and temporally correlated.

The typical super-bubble size is several hundred parsecs, and a large fraction of the superbubble borders on the Galactic-halo medium. The Galactic halo is observationally known to contain hot, tenuous plasma which has similar properties (and possibly the same origin) as super-bubble medium. At a distance of $50 \mathrm{kpc}$ from the Galactic centre the halo density was roughly determined as $10^{-4} / \mathrm{cm}^{-3}[7]$ and from absorption studies the halos of spiral Galaxies are known to extend to several hundred kpc 41].

To good approximation the cannonballs are thus expected to propagate in $85 \%$ of all cases in super-bubble and halo medium and in $15 \%$ of all cases in the normal interstellar medium (with a density of $\approx 0.1 \mathrm{~cm}^{-3}$ ). In addition, in the former case denser material $(\rho$ $\approx 0.1 \mathrm{~cm}^{-3}$ ) from the progenitor star of the $\mathrm{SN}$ in about the first parsec of propagation is expected.

\subsubsection{Cannonball propagation in interstellar medium}

When a mass of magnetised plasma (plasmoid) moves through the ionised interstellar medium two limiting cases can be distinguished.

1. If the plasmoid is impenetrable to the incoming charged particles (e.g. because of a homogenous, strong B field), so that all are effectively reflected, a collisionless bow-shock forms and the particles flow around the plasmoid without any sweep up of ambient matter. 2. If the incoming charged particles can freely enter the plasmoid (e.g.because of a very inhomogeneous B field) no shock forms and all incoming particles are swept up.

Because cannonball B-field is expected to be strong but very turbulent I think an intermediate situation is most likely. The moving cannonball drives an ultrarelativistic collisionless shock into the interstellar medium, but most of the incoming particles (including the ones accelerated at this shock) are eventually swept up by the following cannonball.

\subsubsection{Numerical calculation of cannonball motion}

The following relativistic equations of motion for plasmoids ejected with an initial Lorentz factor of $\Gamma \approx 300$ and an initial energy of $\mathrm{E} \approx 10^{53}$ ergs were numerically integrated using the following expressions 13 :

$$
\begin{array}{r}
d \Gamma / d m=-\left(\Gamma^{2}-1\right) / M \\
d m / d r=\pi R^{2} \rho(r) \\
d M / d r=\Gamma d m / d r
\end{array}
$$

$\mathrm{m}$ is swept up mass, $\rho$ the ambient density and $\mathrm{r}$ the distance travelled by the cannonball. $\mathrm{M}$ is the cannonball mass, and was set to $\mathrm{E} / \mathrm{\Gamma}$ initially. The cannonball radius $\mathrm{R}$ was calculated assuming that the plasmoid expands with a speed of $\mathrm{v}=0.2 \mathrm{c} / \Gamma$ in the observer frame until it reaches a radius of $\mathrm{R}_{\text {final }} \approx 0.3 \mathrm{pc}$. No further expansion was assumed to take place afterwards. $\mathrm{v}$ is similar to the initial expansion in the plasma clouds ejected by GRS 
$1915+105$ and also the speed of sound in a relativistic gas.

As discussed in section 4.3.2 the plasmoid is assumed to sweep up all ambient matter, i.e. it grows in mass. The calculation does not include a treatment of the internal dynamics of the plasmoid, the matter is assumed to move with the plasmoids Lorentz factor at the time of sweep up. Therefore the presented results are only indicative and unrealistic, especially in the final stages of propagation when most of the initial kinetic energy has been transformed into turbulent plasma motion, B fields and CRs.

For the ambient matter the two cases discussed in section 4.3.1 were assumed. In case 1 the cannonball-plasmoid first moves in matter of $0.1 \mathrm{~cm}^{-3}$ for $1 \mathrm{pc}$ (stellar wind), $1 \mathrm{kpc}$ in $10^{-3} \mathrm{~cm}^{-3}$ (super bubble) and then in $2 \cdot 10^{-4} \mathrm{~cm}^{-4}$ (halo). In case 2 the plasmoid moves in matter with a density of $0.1 \mathrm{~cm}^{-3}$ throughout.

Some relevant parameters as a function of plasmoid travel distance are given in figs.6 and 7 .

Case 1 ( $\approx 85 \%$ of all cannonballs, see section 4.3.1)

The plasmoid is slowed down non-ultra-relativistic speeds $(\Gamma<10)$ in the first ca. 40 pc. It then travels for a time $t_{p}$ (some hundreds of thousands years) with subrelativistic speed $(\beta \approx 0.2)$. The number of cosmic-ray producing plasmoids at any time is given as $t_{p} / t_{i}$ where $t_{i}$ is the mean interval of plasmoid producing events (estimated as $50-1600$ years in section 4.1.2). I thus expect some hundreds to thousands of plasmoids in the halo that are still actively accelerating and releasing CRs.

Case 2 ( $\approx 15 \%$ of all cannonballs, see section 4.3.1)

The cannonball Lorentzfactor $\Gamma$ falls below 10 at about $10 \mathrm{pc}$ and its speed $\beta$ below 0.1 at 80 pc. The cannonball does not travel far from its point of origin in a SN, i.e. it remains close to the Galactic centre in general. Consequently also all CRs produced by the cannonball will be released there, within the solar circle.

These results were found to be very insensitive on the assumed initial value of $\Gamma$. However, if the initial energy $\mathrm{E}$ is assumed to be significantly smaller or the final confined plasmoid radius $R_{\text {limit }}$ significantly larger, all cannonballs range out before reaching the Galactic halo.

\section{Acceleration of cosmic rays by and in cannonballs}

\subsection{1st-order Fermi acceleration up to the knee: ultrarelativistic shocks}

Heavens \& Drury [34] - in a pioneering paper on acceleration in relativistic shocks - made the conjecture that the power-law index of particles accelerated in such shocks asymptotically approaches a universal index of $\delta=-2.2$ (eq.(3)) with rising Lorentz factor. This has recently been confirmed by several researchers [39, 4,30 . As this is exactly the source index of the "universal CR sources" in section 2.2, I propose that the bulk of Galactic hadronic CRs is accelerated at the ultra-relativistic shocks driven by cannonballs into the 


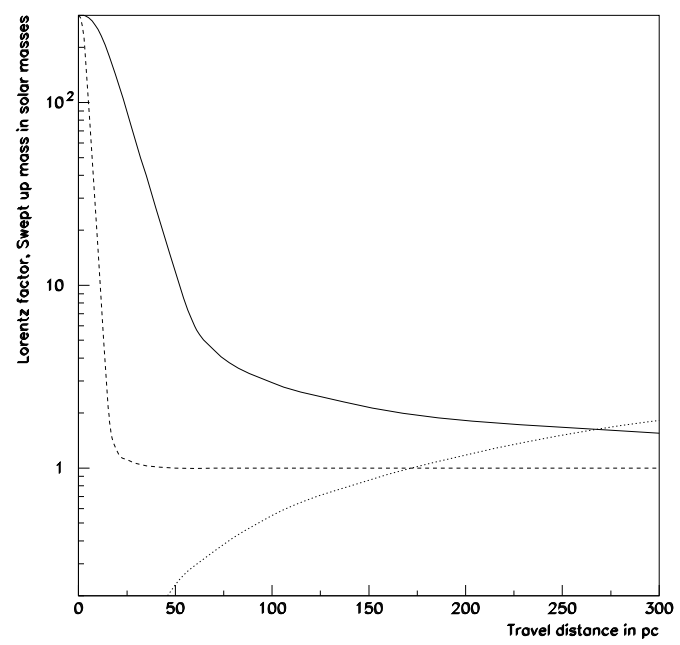

Figure 6: The evolution of the Lorentz factor of a plasmoid with the properties described in the text as function of distance travelled in the observer frame. The full curve is for motion in a tenuous super-bubble medium (case 1), the dashed one for the normal warm interstellar medium (case 2). The dotted line shows the amount swept-up matter in the latter case in units of solar masses for case 2.

interstellar medium. Extragalactic jets, which might be qualitatively similar objects, have been identified as prolific CR accelerators[10], in particular their hot spots [57].

The accelerated particles are immediately swept up into the plasmoid (section 4.3.2) and remain confined there until it has slowed down to subrelativisitic speeds in the Galactic halo (section 5.2). In this scenario a spectrum extending down to small energies with the correct index is expected. The release mainly outside the solar circle leads to a spatial "source distribution" in agreement with the one expected for "universal sources" (section 2.3).

The maximal energy that can be reached in this acceleration process is limited by the extension of the shock and the interstellar magnetic field (the shock propagates in this medium) and is given as [30]:

$$
E_{\max }=3 \cdot 10^{15} \mathrm{eV}(R / 0.02 \mathrm{pc})(Z B / \mu G)(\Gamma / 50)
$$

$R$ is radius of the plasmoid when it has a Lorentz factor of $\Gamma=50$. The preferred value of $R=0.02 \mathrm{pc}$ was chosen based on results of the numerical calculation discussed in section 4.3.3. $\mathrm{Z}$ is the charge of an accelerated particle. A precise calculation of this maximum energy would require a more detailed calculation of the plasmoid dynamics than I have performed, but the inserted numbers are correct to the order of magnitude. I identify $\mathrm{E}_{\max }$ with the "knee energy" $\mathrm{E}_{\text {knee }}($ sect 2.2). The observed knee seems to be a rather "sharp" feature in the primary CR spectrum[2]. In my scenario there are $\approx 1000$ current sources for these CRs. It seems highly unlikely that they would be so uniform in their properties 


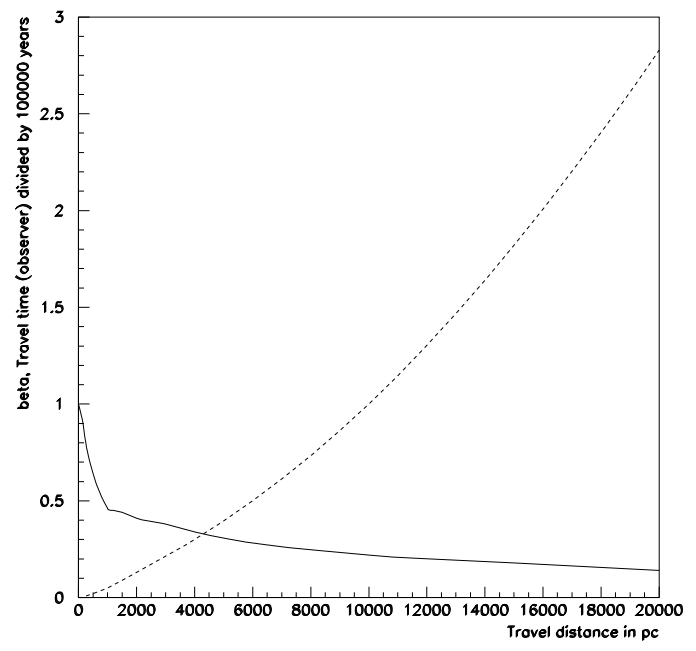

Figure 7: The $\beta=\mathrm{v} / \mathrm{c}$ factor of a plasmoid propagating in a super-bubble environment (case 1) as a function of transversed distance in the observer frame. The dashed curve gives the elapsed time in the observer frame.

that a sharp knee would be a natural featureף. Therefore the sharpness of the knee remains unexplained.

\subsection{2nd-order Fermi acceleration above the knee: the Syrovatskii mech- anism}

While the plasmoids are "coasting" in the super-bubble (case 1 of section 4.3.3) or normal interstellar medium (case 2 ) for a time $\mathrm{t}_{\text {coast }} \approx$ several $10^{5}$ years (case 1 ) resp. hundreds years (case 2) with "subrelativistic" speeds 8 internal turbulent plasma motions will further accelerate the hadronic "swept up" CR pool via the second-order Fermi mechanism. The coasting time scales $\mathrm{t}_{\text {coast }}$ for case 1 and 2 were estimated from the numerical calculation explained section 4.3.3 (see also fig.]).

Without turbulent plasma motion the swept-up CRs remain very efficiently confined in the plasmoid during the coasting phase except at the highest, near-knee energies. The diffusiveescape time scale from a sphere with radius $\mathrm{R}$ and a mean free scattering path $\lambda$ is given as $\mathrm{t}_{d}=1.5 \mathrm{R}^{2} / \mathrm{c} \lambda$ [36]. Here $\lambda$ is a particles mean free path for scattering from magnetic inhomogeneities. I set $\lambda$ to its Larmor radius in this manuscript always (i.e. within the cannonball I always assume the Bohm limit). One then gets for the escape time from the

\footnotetext{
${ }^{7}$ I thank an anonymous referee for pointing this out to me

${ }^{8}$ defined here as $0.2<\beta<0.5$ )
} 
plasmoid with the "confined" radius $\mathrm{R}_{\text {limit }}$ and internal magnetic field strength $\mathrm{B}$ (section 4.2.1):

$$
\begin{array}{r}
t_{d}=10^{5}\left(R_{\text {limit }} / 0.3 p c\right)^{2} \times \\
(B / 0.6 G) /\left(E_{i} / 3 \cdot 10^{15} \mathrm{eV}\right) \text { years } \approx t_{\text {coast }}
\end{array}
$$

At energies below $\mathrm{E}_{\text {knee }} \mathrm{t}_{d}$ exceeds $\mathrm{t}_{\text {coast }}$ by a large margin. The rate of diffusive release which determines the total CR-luminosity (section 4.1.3) - is then determined by the turbulent plasma motion in the plasmoid, and I do not try to determine it here.

It is plausible that equipartition between turbulent motion, magnetic fields and cosmic rays holds in the confined plasmoid. The major energy loss then occurs via loss of cosmic-rays due to diffusion or release in bunches due to disturbances of the magnetic field at the plasmoid boundary. Syrovatskii [64] has shown that in this situation equipartition is restored through particle acceleration and the released CR particles are distributed in energy according to a power-law spectrum with a universal differential index. In particular he shows: At energies that were not previously populated in the initial pool of CRs - i.e. in our case above the "knee" energy - the universal differential power-law index of the released CRs according to eq.(3) is $\delta_{2}=-2.5$.

Moreover, it can be easily shown with the expressions in the appendix of Syrovatskii's paper [64]:

At energies which were populated in the initial pool of CRs with an index of $\delta_{1}=-2.2$ - i.e. in our case below the "knee energy" - the universal differential index of the released CRs is (again) -2.2 . This "invariance" only holds for the index of -2.2 and is thus apparently fortuitous.

Syrovatskii's [64] results are independent of any details of the Fermi acceleration process because they follow from purely thermodynamic considerations.

I thus obtain the source spectrum that we had derived as most likely for the "universal source" class in section 2.2, a power law with an index of -2.2 below the knee and -2.5 above the knee up to the highest observed energies $I$ therefore propose that hadronic CRs mainly stem from cannonballs in the Galactic halo that release previously accelerated particles.

\subsubsection{Possible visibility of Galactic cannonballs in the radio range}

The expected radio luminosity of cannonballs in our Galaxy is rather smaller than one might expect at first sight. In their early ultrarelativistic stage relativistic beaming prevents the visibility of Galactic cannonballs. The emitted radiation is then visible only in external distant galaxies as a GRB afterglow. Galactic cannonballs become in principle visible only in the "coasting phase", when no important beaming takes place and the cannonball accelerates CRs via the Syrovatskii mechanism in the present scenario. In this phase nearly all of the $\mathrm{CR}$ acceleration has already taken place in the present scenario. Only a small fraction 
$f_{2 \text { nd-order }}$ (see below) of the total CR energy produced by a cannonball is accelerated and can contribute to the radio luminosity.

A fundamental uncertainty in predicting the cannonball radio luminosity is the estimation of a factor $\mathrm{f}_{\mathrm{e} / \mathrm{p}}$, giving the CR energy injected into electrons relative to the one injected into nuclei in the coasting phase. Because of the high magnetic field in the cannonball, electrons have a very short lifetime in the plasmoid due to synchrotron losses and need to be injected during the "coasting phase". It is a fundamental assumption of the Syrovatskii mechanism that the injection of new particles from the thermal pool has ceased, so that mainly already-relativistic particles are accelerated [64]. In this limit $\mathrm{f}_{\mathrm{e} / \mathrm{p}}=0$ and the radioluminosity of cannonballs is small. However, it seems likely that this assumption holds only approximately and that $\mathrm{f}_{\mathrm{e} / \mathrm{p}}$ is smaller than 1 by some not extremely large factor. The radio luminosity of a cannonball in the Galactic halo at $1.4 \mathrm{Ghz} \phi_{1.4 \mathrm{GHz}}$ assuming a differential spectral index of the accelrated particles of -2.5 can be parameterized as follows:

$$
\begin{array}{r}
\phi_{1.4 \mathrm{GHz}} \approx 3\left(E_{\mathrm{tot}} / 10^{53} \mathrm{erg}\right) \times \\
\left(R_{\mathrm{prog}} / 1000 \text { years }\right) \times \\
\left(f_{\text {2nd-order }} / 3 \cdot 10^{-5}\right) \times \\
\left(t_{\text {coast }} / 3 \cdot 10^{5} \text { years }\right) \times \\
\left(20 \mathrm{kpc} / d_{\mathrm{CB}}\right)^{2} f_{\mathrm{e} / \mathrm{p}} \mathrm{Jansky}
\end{array}
$$

Here $f_{2 n d-o r d e r}$ is the ratio of the energy injected into CRs in the second-order process relative to the total. The estimated value corresponds to the energy content of CRs above the knee relative to the total because in the present scenario CRs are reaccelerated to energies exceeding the "knee" energy in the "coasting phase" of the cannonballs. $\mathrm{d}_{\mathrm{CB}}$ is the distance of the cannonball.

With a factor $\mathrm{f}_{\mathrm{e} / \mathrm{p}}$ somewhat below 1 the expected radio brightness of cannonballs is in the sub-Jansky regime. A systematic search for nonthermal radio-sources with a high proper motion (on the order of 1 arcsecond/year) is a crucial test of the present scenario. There are 771000 radio sources the recent FIRST catalogue [3] of which only $19 \%$ have optical counterparts. No systematic search for high proper motions was performed in this data base. If one embarks on one, there are many possible technical reasons for false positives 72]. Even at a flux level of one Jansky not all radio sources in the FIRST catalogue are identified. Radio sources with flux levels near 1 Jy are frequently very faint high-z radio galaxies.

In the absence of a proper-motion survey in the radio and/or systematic identifications of all radio sources with a limiting flux level below about a Jansky the absence of "obvious" radio counterparts to cannonballs cannot yet be an argument to reject the present scenario "a limite".

When the cannonball finally stops, it enters Sedov-Taylor phase. In this phase adiabatic losses probably prevent an important release of CRs and its brightness is difficult to predict. For an earlier very preliminary discussion about expected $\gamma$-ray fluxes of cannonballs see 
Ref.55].

\subsubsection{Can second-order acceleration in the plasmoids reach ultra-high ener- gies?}

To reach the highest observed CR energies UH energies (i.e. energy of protons up at least $\mathrm{E}_{f} \approx 10^{20} \mathrm{eV}$ ) in second-order Fermi acceleration three conditions have to be fulfilled [36]. 1. The lifetime of the plasmoid " $t_{\text {coast" ( }}$ (see beginning of section 5.2) has to exceed the time " $\mathrm{t}_{n}$ " necessary for acceleration from the initial $\mathrm{E}_{i}$ to the final energy $\mathrm{E}_{f}$ :

$$
t_{\text {coast }} \geq \text { hundreds of years }>t_{n}
$$

An analogous condition limits the maximum energy that can be reached in CR acceleration in SNR to about $100 \mathrm{TeV}$ [5]. $\mathrm{t}_{n}$ is given as $\ln \left(\mathrm{E}_{f} / \mathrm{E}_{i}\right) \times \mathrm{t}_{a}$. The second-order Fermi acceleration time scale is $\mathrm{t}_{a}=\lambda / 2 \mathrm{c} \beta^{2}[36]$. For a conservative estimate of $\mathrm{t}_{n} \mathrm{I}$ choose the $\lambda$ for the final maximum energy $\mathrm{E}_{f}=10^{20} \mathrm{eV}$.

$$
\begin{array}{r}
t_{n}=400(\beta / 0.5)^{2} \times \\
\ln \left(\left(E_{f} / 10^{20} e V\right) /\left(E_{i} / 3 \cdot 10^{15} e V\right)\right) \times \\
\left(E_{f} / 10^{20} e V\right) /(B / 0.6 G) \text { years }
\end{array}
$$

Taking into account that this estimate is conservative I conclude that the condition (16) can be surely met for protons if $\beta>0.5$.

2. The diffusive-escape time scale $\mathrm{t}_{d}=1.5 \mathrm{R}^{2} / \mathrm{c} \lambda$ must be larger than the second-order acceleration time scale $\mathrm{t}_{a}=\lambda / 2 \mathrm{c} \beta^{2}$ up to the highest energies, otherwise the particles escape before being accelerated. I obtain for protons:

$$
\begin{array}{r}
t_{d} / t_{a} \approx(\beta / 0.2)^{2}\left(R_{\text {limit }} / 0.3 p c\right)^{2} \times \\
(B / 0.6 G)^{2} /\left(E / 10^{20} e V\right)^{2}
\end{array}
$$

The condition is thus fulfilled for $\beta>0.2$.

3. The synchrotron-loss time scale $\mathrm{t}_{s} \simeq \mathrm{k} \mathrm{E}^{-1} \mathrm{~B}^{-2}$ with $\mathrm{k}=\frac{3 m^{4} c^{7}}{2 e^{4} \sin ^{2}(\alpha)}$, has to remain larger than the acceleration time scale $t_{a}$, otherwise acceleration is shut off. $\alpha$ is the pitch angle and is conservatively set to $\pi / 2$ below.

$$
\begin{aligned}
& t_{s}=3.9 /\left(\left(E / 10^{20} \mathrm{eV}\right) \cdot(B / 0.6 G)^{2}\right) \text { years } \approx t_{a} \\
& \approx 3.1\left(\mathrm{E} / 10^{20} \mathrm{eV}\right) /\left((\mathrm{B} / 0.6 \mathrm{G}) \times(\beta / 0.3)^{2}\right) \text { years }
\end{aligned}
$$

The condition is seen to be fulfilled for $\beta>0.3$.

I conclude that there is no basic argument forbidding acceleration of UHE CRs in plasmoids. However, it seems difficult to accelerate protons to energies far above $10^{20} \mathrm{eV}$ with this mechanism. 


\section{Cannonballs as universal CR sources and observational facts}

\subsection{UHE CRs from plasmoids in the Galactic halo: Greisen cutoff and anisotropies on a small angular scale}

If CRs originate in the Galactic halo, the absence of the "Greisen-Zatsepin cutoff" - that is otherwise made quite puzzling by the lack of extragalactic source candidates near the direction of individual UHE-CRs [65] - is readily understood. At a distance of typically 20 kpc no significant absorption of UHE protons in the $3 \mathrm{~K}^{\circ}$ background radiation is expected. There is some evidence for a small-scale clustering in the arrival directions of UHE CRs [67. The number of discrete sources necessary to explain the observed degree of clustering was estimated as $\approx 400[24]$ in agreement with the order of magnitude number of cosmic-ray producing plasmoids estimated in section 4.3.3. Consequently it is proposed to identify the subrelativisitic cannonballs in the Galactic halo as the sources responsible for the small-scale clustering 19].

\subsection{Expected anisotropy of CR arrival directions from coasting cannon- balls}

\subsubsection{At energies below the ankle}

Core-collapse supernovae are well known to occur predominantly within the solar circle [5]. Therefore those cannonballs that range out in the dense disk-interstellar medium (corresponding to case 2 in section 4.3.3) release all CRs at Galacto-centric distances smaller than the solar one. These CRs (estimated to be about $15 \%$ of the total in section 4.3.3) will contribute to the anisotropy of CRs at all energies. We had seen in section 3.2 that about this fraction $f$ (Eq.7) of CRs is required to be released by the "universal" sources within the solar circle. The cannonballs therefore remarkably "fit the bill" derived for "universal sources"in section 3.2 .

\subsubsection{At energies above the ankle}

The absence of significant large-scale anisotropies of arrival directions at UHE energies (section 2.3.2) is understood because an observer inside an emitting sphere sees no anisotropies. However about $15 \%$ of all cannonballs are expected to release CRs at Galactic locations similar to the ones of SNe (section 4.3.3). Thus it is predicted that about $15 \%$ of all CRs that propagate roughly on straight lines in the Galactic magnetic field (i.e. with energies well above the ankle) come from the general directions of the Galactic centre and disk. Presently the world-data sample at these energies is not large enough to confirm or rule out this prediction. 


\subsection{Galacto-centric distribution of the CR density}

I had discussed in section 3.1 that the very small observed gradient of CR density as a function of Galacto-centric radius strongly points towards CR sources mainly outside the solar circle. The cannonballs are expected to release $\approx 15 \%$ of all CRs within the solar circle (section 4.3.3), so a small non-vanishing gradient is expected. The data are in qualitative agreement with this expected "cannonball density distribution" but the presence of large systematic errors seem to make a further detailed test of the scenario in this area difficult.

\subsection{Predicted chemical composition of Cosmic Rays at all energies}

One can now calculate the chemical composition of cosmic rays. At low energies, below the knee, CRs are accelerated from the local interstellar medium and are thus expected to have abundances at a given energy per nucleus similar to the solar ones, as observed. I take the observed abundance at low energies as the starting point. In both first and second order Fermi acceleration identical power-law indices for all nuclei are expected. The knee (eq. (13)) and ankle positions in energy are proportional to Z. With these assumptions the spectrum for all indices can be predicted (fig8). We saw in section 5.2.2 that acceleration of protons beyond $\times 10^{20} \mathrm{eV}$ is difficult in cannonballs and therefore assume an exponential cutoff at an energy/nucleus above $\mathrm{Z} \cdot 10^{20} \mathrm{eV}$. It is seen that in the universal scenario the chemical composition gets heavier near the knee - similar to what is expected in many other models. Near the ankle the composition becomes very light, similar to the expectations in some extragalactic scenarios for the origin of UHE CRs but finally, beyond $10^{20} \mathrm{eV}$ the composition becomes heavy again.

\section{Discussion}

\subsection{Assumptions}

The major assumptions of the present model for CR origin are:

1. The basic features of the "cannonball model" for GRBs [20] are correct.

2 . The CR propagate diffusively in a relatively large Galactic halo (radius $\approx 20 \mathrm{kpc}$ ). The energy dependence of the diffusion coefficient is Kraichnanian at all energies.

3. The total energy of the ejected cannonballs is $\approx 10^{53} \mathrm{ergs}$, about an order of magnitude higher than often assumed.

4. After an initial expansion the cannonball remains confined to a radius of about $0.3 \mathrm{pc}$, due to ram-pressure and magnetic effects until it has slowed down to speeds below about $0.1 \mathrm{c}$.

While there are theoretical and observational arguments in favour of assumption 1 and 2, assumptions 3 and 4 have no better motivation presently than to make the model work. Additional minor assumptions are discussed in the text. 

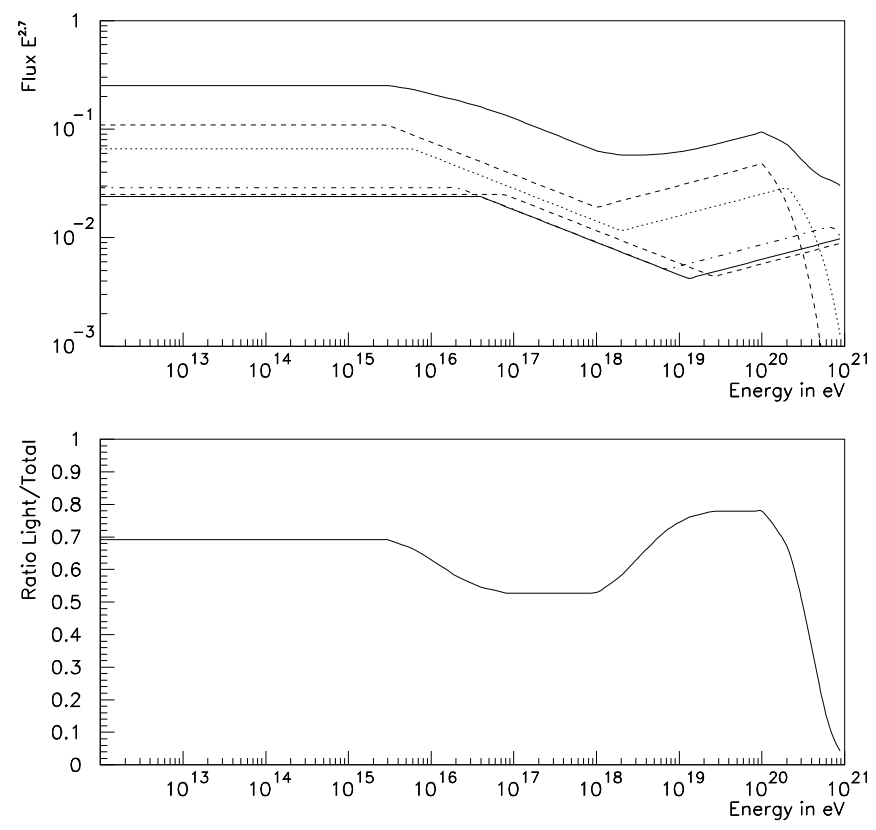

Figure 8: Upper panel. The intensity of 5 chemical groups (in the order of falling intensity at low energies: p (dashed),He (dotted),CNO group (dash-dot), Ne-S group (dashed), Cl-Fe $\operatorname{group}\left(\right.$ full)) in $\mathrm{m}^{-2} \mathrm{~s}^{-1} \mathrm{sr}^{-1} \mathrm{TeV}^{1.7}$ as a function of energy/nucleus. The uppermost line is the sum of all components in the same units. The composition for low energies below the knee is taken from Wiebel[74]. Above the knee the prediction of the present model is shown. Lower panel: the ratio of the light element intensity $(p+\mathrm{He})$ to the total intensity. as derived from the upper panel.

\subsection{Achievements}

The location of CR sources at all energies mainly in the Galactic halo and with a fraction $f$ $\approx 15 \%$ near the Galactic centre expected in our model allows to understand the following observations more naturally than many other models:

1. The absence of the Greisen-Kuzmin cutoff together with the absence of a large scale anisotropy at energies above about $4 \times 10^{19} \mathrm{eV}$ and extragalactic counterparts in the direction of the highest-energy CRs.

2. The observed anisotropy of $\mathrm{CR}$ with energies between about $100 \mathrm{TeV}$ and $10^{18} \mathrm{eV}$ quantitatively.

3. The Galacto-centric distribution of low-energy hadronic CRs inferred from $\gamma$-ray astronomy.

The origin of hadronic CRs in about 1000 cannonballs speeding through the interstellar and interhalo medium allows to understand:

4. The possible indication for small-scale clustering is understood as being due to emission from individual cannonballs. 
5. The origin of the "knee feature" in the hadronic CR spectrum and the form of the source-spectrum and higher and lower energies. The observed "sharpness" of the knee remains unexplained, however.

6. Finally the idea of a "universal-source class", accelerating CRs at all energies, leads to a very natural understanding of the energy for the "ankle" feature in the spectrum of Galactic CRs.

\subsection{Predictions}

The most characteristic prediction of a universal origin of hadronic CRs at all energies is the variation of chemical composition above the knee shown in fig.(8). Evidence for a Kolmogoroff rather than Kraichnan energy dependence of the diffusion coefficient for cosmic rays (e.g. from the ongoing experimental studies on reacceleration) would rule out the "universal scenario" presented here. If "cannonballs" accelerate all CRs above the ankle, on the order of 1000 spatially discrete sources of UHE CRs must be found by the next generation of UHE-CR detectors. About $15 \%$ of these clusters must be preferentially spatially distributed like core-collapse SNe (i.e. near the Galactic disk and centre).

The counterparts of these sources must be very compact plasma clouds with subrelativistic proper-motions. The present model stands or falls with the existence of counterparts to the cannonballs in various regions of the electromagnetic spectrum.

Acknowledgements I thank Maria Diaz for reading the manuscript and Arnon Dar and Alvaro De Rújula for many helpful explanations of the cannonball model. I am grateful

to an anonymous referee for helpful criticism. The author is supported by a Heisenberg fellowship of the DFG.

\section{References}

[1] Armstrong J.W., Cordes J.M., Rickett B.J., 1981. Density power spectrum in the local interstellar medium, Nature 291,561-564.

[2] Arqueros et al. (HEGRA collaboration), 2000. Energy spectrum and chemical composition of cosmic rays between 0.3 and $10 \mathrm{PeV}$ determined from the Cherenkov-light and charged particle distribution in air showers, A \& A 359,682-694.

[3] Becker R.H., et al., 2001. FIRST, http://sundog.stsci.edu

[4] Bednarz J., Ostrowski M., 1998. Energy Spectra of Cosmic Rays Accelerated at Ultrarelativistic Shock Waves, PhRvL 80,3911-3914.

[5] Berezinskii V.S., Bulanov S.V., Dogiel V.A., Ginzburg V.L., Ptuskin V.S. , 1990. Astrophysics of cosmic rays. North Holland, Amsterdam. 
[6] Biermann L., Davis L., 1958.On the origin of Cosmic Rays during the early part of the Evolution of our Galaxy, Z.Naturforsch. 13a,909-915.

[7] Biermann P.L., 1995. The origin of cosmic rays, PhRvD 51,3450.

[8] Bloemen H., 1993. Diffuse Galactic Gamma-Ray Emission Above $1 \mathrm{MeV}$, AIP Conf. Proc. 278,409.

[9] Burbidge G., 1955. Halo of radio emission and the Origin of Cosmic Rays, Phys.Rev. 101,906-907.

[10] Burbidge G., 1962. The origin of Cosmic Rays, Prog. Theoret. Phys. 27,999.

[11] Cen R.,1998, Supernovae, pulsars and gamma-ray bursts: a unified picture. astroph/9809022, ApJL.

[12] Cesarsky C.J.,1980. Cosmic Ray propagation in the Galaxy, ARA\&A, 18, 289-319.

[13] Chiang J., Dermer C.F., 1998. Synchrotron and SSC Emission and the Blast-Wave Model of Gamma-Ray Bursts, astro-ph/9803339.

[14] Clay R.W., Donough M.-A., Smith A.G.K., Dawson B.R., 1998. Anisotropies and the power requirements for Galactic Cosmic Rays, PASA 15, available at http://www.atnf.csiro.au/pasa/15 $2 /$

[15] Compton A.H., Getting I.A., 1935. An apparent Effect of Galactic Rotation on the Intensity of Cosmic Rays, Phys.Rev. 47,817-821.

[16] Connell J.J., Simpson J.A., 1999. Testing the role of Cosmic Ray reacceleration, Proc. 26th ICRC,vol.3,p.33-26.

[17] Dar A., 1998. Cosmic rays and gamma-ray bursts from microblazars, astroph/9809163.

[18] Dar A., 1999. Are extragalactic Gamma Ray Bursts the Source of the Highest Energy Cosmic Rays?, astro-ph/9901005.

[19] Dar A., Plaga R., 1999. Galactic $\gamma$-ray bursters - an alternative source of cosmic rays at all energies, A\&A 349,259.

[20] Dar A., De Rújula A., 2000a. A cannonball model of gamma-ray bursts: superluminal signatures, astro-ph/0008474, A \& A in the press.

[21] Dar A., De Rújula A., 2000b. The Cannonball Model of GRBs: Temporal and Spectral Properties of The Gamma Rays, astro-ph/0012227, submitted A \& A. 
[22] Dermer C., 2000. Neutrino, Neutron, and Cosmic Ray Production in the External Shock Model of Gamma Ray Bursts, astro-ph/0005440, published sparately in: ApJ 574 in the press; and ApJ 556, 479.

[23] De Young D.S., Axford W.I., 1967. Inertial confinement of Extended Radio Sources, Nature 216,129.

[24] Dubovsky S.L., Tinyakov P.G., Tkachev I.I.,2000. Statistics of clustering of ultra-high energy cosmic rays and the number of their sources, astro-ph/0001317, Phys.Rev.Lett. $85,1154$.

[25] Erlykin A. D., Wolfendale A.W., Zhang L., Zielinska M., 1996. The gradient of cosmic ray protons in the outer Galaxy, A\&ASS 120,397.

[26] Erlykin A. D., Wolfendale A.W., 2000. The origin of PeV cosmic rays, A\&A 356,L63L65.

[27] Fenimore E.E., Ramirez-Ruiz E., 2000. Redshifts for 220 BATSE Gamma-Ray Bursts Determined by Variability and the Cosmological Consequences, astro-ph/0004176.

[28] Fishman, G. J. \& Meegan, C. A. A. 1995. Gamma-Ray Bursts, ARA\&A 33,415.

[29] Fryer C.L., Woosley S.E, 1999. Formation Rates of Black Hole Accretion Disk Gamma-Ray Bursts, astro-ph/9904122.

[30] Gallant Y.A., Achterberg, A.,1999. Ultra-high-energy cosmic ray acceleration by relativistic blast waves astro-ph/9812316 v2, MNRAS 305, L6.

[31] Ginzburg V.L., 1957. The origin of cosmic rays, Prog. Elem. Particle Cosm. Ray Phys. 4,339

[32] Ginzburg V.L., Ptuskin V.S., 1976. On the origin of cosmic rays: Some problems in high-energy astrophysics, RvMP 48,161-189.

[33] Hayashida N. et al. (AGASA coll.),1998. The Anisotropy of Cosmic Ray Arrival directions around $10^{18} \mathrm{eV}$, astro-ph/9807045, $\mathrm{APh} 10,303-311$.

[34] Heavens A.F., Drury, L.OC.,1988. Relativistic shocks and particle acceleration, MNRAS 235,997-1009.

[35] Heinz S., 2000. Beams, Bursts, Bubbles and Bullets: Relativistic Outflows in Astrophysics, $\mathrm{PhD}$ thesis University of Colorado, chapter 5.6.3, http://jilawww.colorado.edu/www/sro/thesis/heinz/index.htm

[36] Hillas A.M., The origin of Ultra-High-Energy Cosmic Rays, 1984. ARA \& A, 22,425444. 
[37] Hunter S.D et al., 1997. EGRET Observations of the Diffuse Gamma-Ray Emission from the Galactic Plane, ApJ 481,205.

[38] Kembhavi A.K., Narlikar J.V.,1999. Quasars and active galactic nuclei. Cambridge University Press, Cambridge.

[39] Kirk J.G., Guthmann A.W., Gallant .A., Achterberg, A.. 2000. Particle acceleration at ultrarelativistic shock: a eigenfunction method, astro-ph/0005222, ApJ 542,235.

[40] Lamb, D.Q., 2000. Implications of recent observational dicoveries for the nature and origin of gamma-ray bursts, astro-ph/0005028.

[41] Lanzetta K.M., Bowen D.V, Tytler D.,Webb J.K., 1995. The Gaseous Extent of Galaxies, ApJ 442,538-568.

[42] Lilly, S.J. et al. 1996. The Canada-France Redshift Survey: The Luminosity Density and Star Formation History of the Universe to Z approximately 1, ApJ 460, L1.

[43] Lingenfelter R.E., Higdon J.C, Ramaty R., 2000. Cosmic Ray Acceleration In Superbubbles ad the Composition of Cosmic Rays, astro-ph/0004166.

[44] Linsley J., 1983. Spectra, anisotropies and composition of cosmic rays above 1000 GeV, Proc. 18th ICRC Bangalore 12,135.

[45] Longair M.S.,1997. The high-energy radiation of active galactic nuclei, In: G. Münch et al. (Ed.), The Universe at Large. Cambridge University Press, Cambridge, p.218.

[46] Longmire C.L.,1963. Elementary Plasma Physics, Interscience (New York, London) p.68-72.

[47] Loveday, J. et al. 1992. The Stromlo-APM Redshift Survey. I - The luminosity function and space density of galaxies, ApJ, 390, 338.

[48] Milgrom M., Usov V., 1996. Gamma-ray bursters as sources of cosmic rays, Astropart. Phys. 4,365.

[49] Morrison P., 1957. On the origins of Cosmic Rays, RvMP 29,235.

[50] Nagano M., Watson A.A., 2000. Observations and implications of the ultrahighenergy cosmic rays, RvMP 72,689-732.

[51] Paczyński B., 1997. Are gamma-ray bursts in star forming regions?, astroph/9710086, ApJ 494,L45.

[52] Paczynski B., 1999. Gamma-Ray Burst - Supernova Relation, astro-ph/9909048, In: M. Livio, N. Panagia and K. Sahu (eds.), Proc. of the Space Telescope Science Institute 1999 May Symposium: "Supernovae and Gamma Ray Bursts; The Largest Explosions Since the Big Bang". Cambridge University Press, Cambridge, p.1. 
[53] Plaga R., 1998. An extragalactic "flux trapping" origin of the dominant part of hadronic cosmic rays? A \& A 330,833.

[54] Plaga R., 2001. The cepheid-like relationship between variability and luminosity explained within the "cannonball model" of Gamma-Ray bursts, astro-ph/0012060, A\&A $370,351$.

[55] Plaga R.,de Jager O. \& Dar A., 1999. Are Galactic Gamma-Ray Bursters the Main Source of Hadronic Non-Solar Cosmic Rays at all Energies? Proc. 26th ICRC, Salt Lake City, 4,353.

[56] Pugliese G., Falcke H., Wang Y. \& Biermann P.L., 2000. The jet-disk symbiosis model for Gamma Ray Bursts: cosmic ray and neutrino background contribution, astro-ph/0003025, A\&A 358,409.

[57] Rachen J., Biermann P.L., 1993. Extragalactic ultra high energy cosmic rays, I. Contribution from hot spots in FR-II galaxies, A\&A 272,161.

[58] Ramirez-Ruiz E., Fenimore E.E., 1999. Pulse width evolution in gamma-ray bursts: evidence for internal shocks, astro-ph/9910273, ApJ 539,712.

[59] Scalo J., Wheeler J.C., 1999. Astrophysical and Biological Implications of Gamma Ray Burst Properties, astro-ph/9912564.

[60] Schmele D., 1998. Suche nach räumlichen Anisotropien der kosmischen Strahlung im Energiebereich oberhalb von $20 \mathrm{TeV}, \mathrm{PhD}$ thesis, University of Hamburg, available on http://www.desy.de/ schmele/DOKTOR/

[61] Spitzer L., 1990. Theories of the hot interstellar gas, ARA\&A 28,71-102.

[62] Strong A.W., Mattox J.R., 1996. Gradient model analysis of EGRET diffuse Galactic gamma-ray emission, A\&A 308,L21.

[63] Strong A.W., Moskalenko I.V., 1998. Propagation of Cosmic-Ray Nucleons in the Galaxy, astro-ph/9807150v2, ApJ 509,212.

[64] Syrovatsky S.I., 1961. Spectrum of Galactic and Solar Cosmic Rays, Sov.Phys. JETP 13,1257-1260.

[65] Takeda M. et al. (AGASA coll.),1998. Extension of the Cosmic-Ray Energy Spectrum Beyond the Predicted Greisen-Zatsepin-Kuzmin Cutoff, Phys.Rev.Lett. 81,1163; astro-ph/9807192.

[66] Tkaczyk W., 1995. The Cosmic Ray Spectrum and irregularities in the Galactic magnetic field, Proc. 24th ICRC, Rome, 3,293. 
[67] Uchihori Y., Nagano M., Takeda M., Teshima M., Lloyd-Evans J. \& Watson A.A., 1999. Cluster Analysis of Extremely High Energy Cosmic Rays in the Northern Sky, astro-ph/9908193, APh 13,151-160.

[68] Vietri M., 1995. On the acceleration of Ultra High Energy Cosmic Rays in Gamma Ray Bursts, ApJ 453,883.

[69] Wang L., Howell D.A., Höflich P., Wheeler J.C., 1999. Bi-polar Supernova Explosions, astro-ph/9912033.

[70] Waxman E., 1995. Cosmological Gamma Ray Burst and the highest energy Cosmic Rays, PhRvL 75,386.

[71] Weiner B.J., Williams T.B., 1999. Detection of $\mathrm{H} \alpha$ Emission from the Magellanic Stream: evidence for an Extended Gaseous Galactic Halo ,AJ 111,1156-1163.

[72] White R.L., 20001. Private communication, January 5.

[73] Wibig T., Wolfendale A., 1999. The anisotropy of arrival directions of cosmic rays above $10^{17} \mathrm{eV}$, J.Phys.G 25,2001-2009.

[74] Wiebel B., 1994. Chemical composition in high energy cosmic rays, Report WUB 94-08, available on http:// wpos6.physik.uni-wuppertal.de:8080 /Public/papers-public.html

[75] Woosley S.E.,1993. Gamma-ray bursts from stellar mass accretion disks around black holes, ApJ 405,273. 